\title{
The "narrative menu" as a publishing channel to disseminate information on food
}

\author{
Raoul Ciappelloni ${ }^{{ }^{*}}$ \\ ${ }^{1}$ e-Journal Sanità Pubblica Veterinaria SPVet.it (http://spvet.it/ - ISSN 1592-1581) Perugia, Italy
}

\begin{abstract}
In this work, an advanced version of the standard restaurant menu, here named as "narrative menu", is presented. A narrative menu is a smart periodical publication containing selected information on food. Through this restaurant table accessory, diners can access several information on food production and cooking, such as texts, videos, podcasts, datasets. This functionality can be achieved through the interaction between customer smartphones and the augmented pages of the menu, provided with insertions such as QR codes, NFC microchips, shortened links or virtual reality triggers. Narrative menu is an advanced publication using an atypical distribution channel. Instead of being available at newspaper kiosks or bookshops, it is available in restaurants, cafeterias and snack bars. Through the smartphone, customers can get information on the food they are about to eat: for instance, characteristics of ingredients, product origin and cooking methods. This kind of smart publication is intended to play a key role in improving the customer's understanding of the gastronomic, cultural and nutritional value of the restaurant's dishes. In addition, narrative menus can also improve the economic return of the catering and food producers, with positive effects also on the territories where they are produced. The initiative can represent the best investment that food producers can make to properly inform their customers on the gastronomic value of proposed foods.
\end{abstract}

\section{Introduction}

The menu is a key factor in the communication between food providers and customers and gives them a first grasp about dishes and restaurant quality. This study would contribute on promoting and enhancing a specialized "Food Storytelling" through alternative editorial distribution channels, based on "augmented" versions of the ordinary paper menus of restaurants, cafeterias and snack bars.

The motivation of this work, linked to the communication needs, arises from the interaction among consumers, food preparers, dealers and food producers.

It is assumed that in the past there was a better interaction between these subjects, typical of non-globalized societies, where city-countryside connections are more direct. 
At present, agri-food initiatives are promoted as sustainable systems, following different strategies, from specific marketing approaches to the creation of computer networks $[1,2,3,4]$.

This "new alliance" between food consumers and food providers is the base of the narrative menu we are proposing and part of a new circular economy business model.

The work originates from the research made by the journal Veterinary Public Health (http://spvet.it) on augmented wall newspaper and scientific smart posters [5,6,7].

The approach was discussed during a series of public meetings held from 2009 to 2020 , based on activities carried out within the "Working Group for biomedical narrative" (2009) [8]. Particularly, menu-like publications were the central themes of a series of theatre lectures, a project funded by the Italian Ministry of Education and presented at the 14th European Association for Health Information and Library Congress (Rome, 2014) [9].

Augmented printed surfaces (in the form of "smart posters") have been discussed in library lectures (2015), within the Didactic Veterinary Hospital of Perugia University, Italy (OVUD, 2016) [10], to introduce students to the use of smart posters for food communication.

From the customers' perspective, a menu is just a set of printed sheets with a list of available dishes, proposed by the restaurant or food provider. Descriptions show the main ingredients, prices and, occasionally, pictures. Regular menus at the most may contain just good design characteristics and pricing strategies [11, 12].The menu we are proposing is structured in a different way, and is mainly used to recall information. This "augmented" version of the paper menu is equipped with short communicative texts (microfiction), graphics, NFC microchips, QR Code tags, shortened links, augmented reality markers or triggers. These technologies may be applied through smartphones or tablets and may give access to information resources via Web or specific repositories on food, with selected scientific literature.

Thanks to the smart menus, during lunch or dinner time, customers may activate a multilevel document delivery system, in order to access information and services of interest on food and gastronomy.

This digital publishing channel may also be used by customers to start relationships (interactions) with privileged witnesses, such as restaurateurs, restaurant managers, food and beverage directors and cooks, but also farmers and food processors, embracing local territories and culture, transforming a simple meal into a cultural experience.

Last but not least, a narrative menu represents a form of entertainment, providing links to games, tests and quizzes on food. In this way, the restaurant environment will be qualified and suitable not only for eating, but also for exchanging knowledge and establishing social relationships.

Potential users of narrative menus are the tourists and all consumers who approach foods they do not know at all and who are interested in learning more or deepening their awareness. The business area of this alternative publishing activity is outlined in Figure 1. 


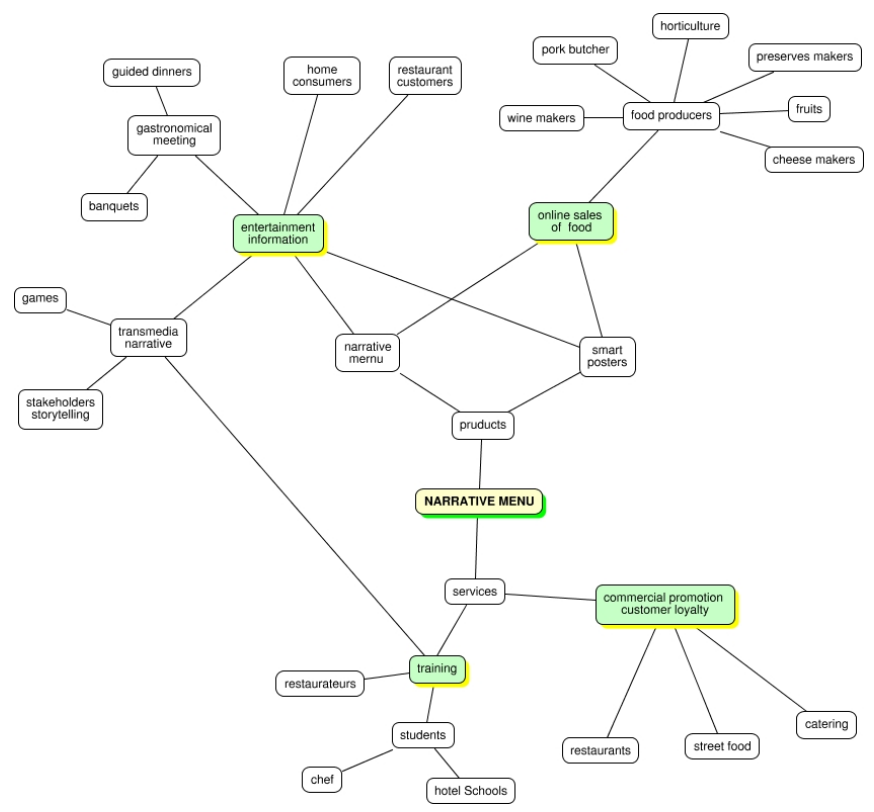

Fig. 1. The structure of the narrative menu publishing initiative and the basic fields of interest: information and entertainment; customer loyalty; sales of food and commercial promotion.

The main actors involved in the issue are customer-readers, restaurateurs, publishers and food manufacturers. These subjects may use the narrative menu for different purposes:

- Customer-readers. They use the smart menu mainly as a convenient source of information on food they are about to consume, to entertain themselves (and diners) while waiting for the dishes and as a catalogue of products to buy online, for their needs;

- Restaurateurs. They actively collaborate with publishers in providing the information they want to convey through the narrative menu, with the purpose to focus the attention of their customers on their gastronomy and qualified products. Through the narrative menus, they can offer a new tool to the staff to suggestively explain dishes and wines. Restaurateurs can also take advantage offering take-away services and ready-to-cook preparations;

- Publishers. For publishers, the menu/smartphone system represents an atypical distribution channel for the promotion of specific transmedia literature on food and food producers. They collaborate with restaurateurs and staff involved in food preparation and distribution, for the management of the editorial calendar and diffusion of specific publications;

- Food manufacturers. For them, the menu based on storytelling is a convenient ecommerce tool to directly and effectively contact selected $\mathrm{n}$ ew potential customers. This function is of interest for some traditional and niche productions, such as cured meats, cheeses and wines.

\section{Inside the narrative menu}

So far, informative exchange through a menu, has been at the centre of attempts based on different conceptions. One of these is based on labeling and indicating food composition. It represents a low-cost education strategy for consumers, in order to foster a responsible food choice $[13,14,15]$. 
The commercial communication to consumers through the menu is another basic topic in this area $[16,17]$.

Computers and digital interfaces to deal with waiters or chefs, have been applied in several systems $[18,19,20]$, in the attempt to transpose a standard restaurant menu structure directly in a tablet or smartphone screen, like in Jotform ${ }^{\circledR}$ Food Ordering or in MyMenu ${ }^{\circledR}$ Systems.

Some strategies have been the subject of patenting, in order to organize an interactive restaurant communication system via intelligent terminals placed on the table [21].

Also the direct contact and human sharing is getting new attention in the restaurant industry and it will certainly grow over time [22]. Among the main needs expressed by consumers, the straightway information usability and the accessibility of multimedia resources, are important topics. Interactive communication tools meet these requests, connecting consumers to information about food and related production processes [23].

It is not yet frequent to use restaurant menus as a literary work, as pamphlets printed not only for providing to customer a list of available food items, but also to share a storytelling experience and entertainment tied to the stories, cultures, landscapes and the beauty behind them $[24,25,26]$.

In this context, our narrative menu has a role to play and some innovative solutions. In comparison to other similar systems, following the Bolter and Grushin conception (1999) [27], it represents a "remediation" of an analogic publication that simulates a digital one.

Starting from this assumption, a new menu organization can be outlined. In our case, we are concerned with the communicative organization of the menu following the metaphor of a window, opened on the local agricultural products and the people involved with them.

These stories, often about traditional non intensive production methods, involve farmers, cheese-makers, butchers, wine producers and all other food manufacturers, allowing us to deal with attractive descriptions at a communicative and narrative level.

It stimulates the reader's (diner's) interest and curiosity, sustaining an advanced form of marketing, based on gastronomic culture and sustainable agriculture systems.

\section{Menu Structure}

The narrative menu can feature as a new editorial strategy to sustain initiatives of publishers aimed to find unconventional fields for transmedia storytelling and electronic commerce services for diners regarded as readers.

In this way, we have two distinct layers in the narrative menu organization:

- the information technology (IT) shell. Hardware and software interface, capable to handle access, storage and distribute, narrative contents;

- an editorial organization, structured to interact with the restaurant team, in order to elaborate literature in a convenient format to be enjoyed in a limited time span.

For the IT shell, through the insertion in the printed surface of "sensitive areas", that can interact with smartphones and tablets, it is possible to access an extemporary "document delivery" of various resources concerning recipes, specific food info and food masters short stories.

An example of the organization of a narrative menu is depicted in Figure 2, regarding a sample page (appetizers). All dishes listed are provided with a suite of devices that make the paper menu connected with other resources that guests can select and download: first of all, a short description and contextual images (dish anatomy, with schemes and labels). QR Codes and shortened links pointing to selected web resources are disposable.

For particular purposes, also Near Field Communication (NFC) microchips for instant smartphone interaction can be inserted, especially in the cover of the menu. Augmented reality triggers may be positioned in different places (close to titles, introducing specific arguments, or special descriptions of dishes), particularly interesting for future developments. 
For instance, augmented reality is excellent to quickly show and manipulate 3D objects and short animations.

At the beginning of this experience, we experimented with the Aurasma - HP Reveal system. To test the VR functionality, other systems are available, like cxocARd (the new version of Aurasma -https://cxocard.com/) or MAXST Systems (http://maxst.com).

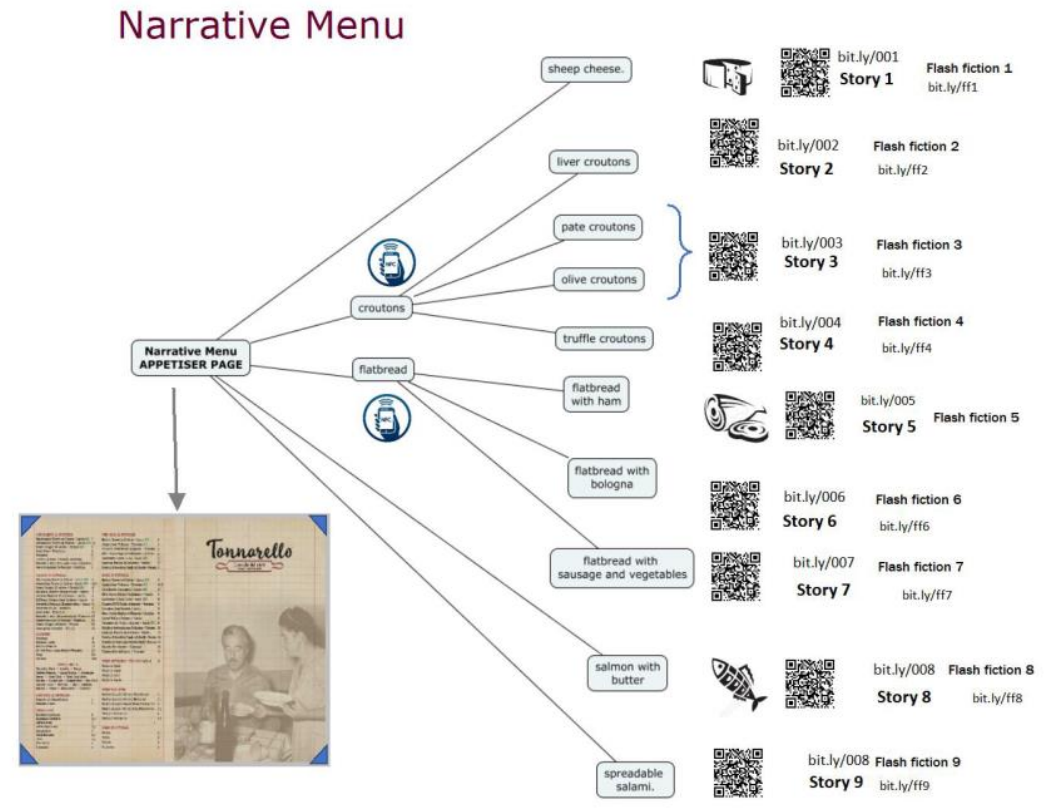

Fig. 2. Like in a scientific smart poster, the "augmented" version of a menu (short communicative texts - micro-fiction and graphics aside), makes use of four components: NFC microchips, QR Code Tags, shortened links, augmented reality triggers. It can bring at restaurant and take-away level, a wealth of storytelling from people involved in food preparation. This gives to a professional editor the opportunity to set up an editorial series of essays whose main topic is "people and products" coming from local agro-ecosystems linked to the restaurant business.

The cloud side of the narrative menu interface can be a very light system, based on SaaS platform (hosting web server, database, eCommerce) and associated suite of Apps. In order to foster these initiatives, an open copyright policy should be adopted, based on the Creative Commons licenses suite. For derivative publishing products and implementation protocols, GNU Free Documentation License and derivatives can be adequate. 


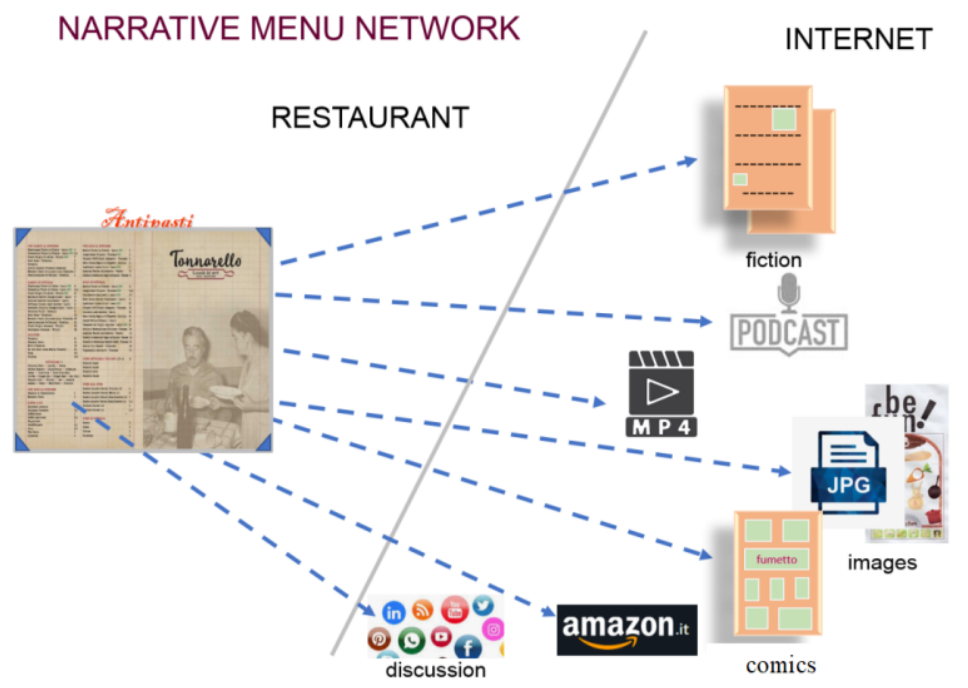

Fig. 3. Structure of a narrative menu page, with the system outputs evidenced.

The adoption of smart menus in a restaurant produces a whole range of new communication opportunities, thanks to a very light editorial organization (Indie or integrated in the restaurant administration), in collaboration with personnel staff (as data collectors and peer reviewers), storytelling writers and interested stakeholders.

Publishers have to face with some particular issues. Firstly, the narrative menu must help customers' choice and help them understand what they eat, avoiding to make these decisions just more complex and time consuming.

Secondly, in order to set up an effective narrative menu structure, it is crucial taking into account the customer behaviour and reading preferences.

For this reason, it is necessary to monitor the customer reaction. The restaurant staff, in connection with the publishing team, may carefully evaluate the time that customers dedicate to the narrative menu and the true impact on customers. It is also important to find a confidential ways to interact with customers at the table, to give them a simple user training on the smartphone-narrative menu usage. A fair observation of customers' preferences can be also performed through the analysis of the menu system logs.

The profiling of users may provide data on individual preferences for dishes and beverages, evaluating the time spent with the proposed storytelling, frequency of videos or images downloads, interaction with other customers on menu topics, access to e-commerce services and contacts with food suppliers. This dataset can be relevant for both restaurants and publishers.

\section{A reference structure of the food narrative}

In order to set up the storytelling for each dish reported in the smart menu, the narration must be organized in a sequence of block structures.

The vector of records (five blocks or chapters) defines a composite narrative space, ideally containing the storytelling that goes "from field to dish".

Block-chapters for a single narration in the menu are as follows:

1 People narration chapter - At the beginning, the food product and its territory is described, coming into the scene with short stories of people involved in the production of ingredients, dairy products, butchery food, etc.; 
2 Kitchen chapter - description: birth of a dish. Through the combination and manipulation of ingredients in the kitchen, the gastronomic preparation is visually described;

3 Dish chapter - description: the dish is ready. Plating, shape, presentation, combinations and presence of other ingredients in the preparation, are illustrated;

4 Wine chapter - description: wine relationships. The combination of dishes with wines and beverages is narrated. Epilogue of the story;

5 Synthesis chapter - description: Restaurant staff enters in the scene as storytellers. Using all menu resources, they guide and encourage the customer's choices.

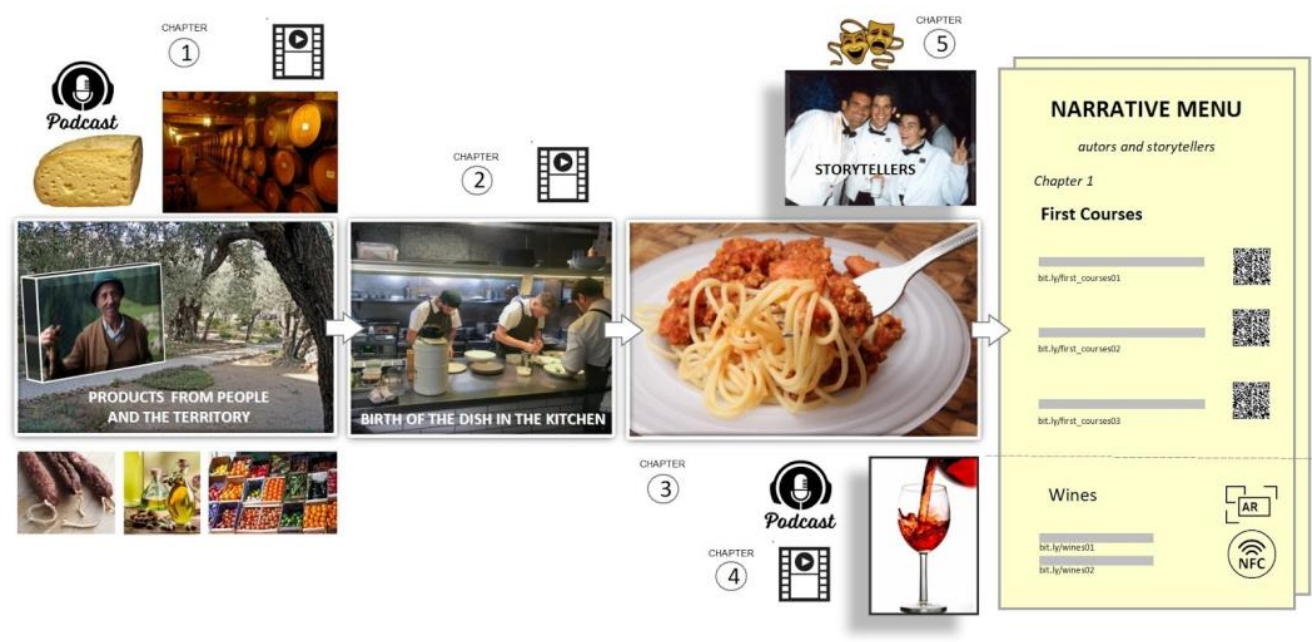

Fig. 4. Contents of a narrative menu: Each narration is dealing with people and products of the territory, combination of ingredients, plating and presentation, pairing with wines and beverages.

This ideal sequence, with some variations, is staged in the restaurant, simulating a theatrical performance based on narrative menu contents.

This performance must meet some requirements:

- Information must not concern food in a general sense, but specifically those the consumers are going to deal with. This is the most important requirement;

- The descriptive texts must be short and evocative (inspired to flash fiction), easily readable on a smartphone screen. Duration of video clips should not exceed one minute (the "one minute madness" approach should be applied, as in some congress communications or in scientific poster sessions);

- Texts on the narrative menu should have some style and literary value, not only be informative;

- The sound level of video and podcast components must be limited, in order do not represent a nuisance for other customers;

- There must be the possibility to criticize narrations and videos displayed, leaving opinions or guest reviews in the system;

- If possible, the information on food should be integrated into entertainment products, such as serious games (food games or quiz/tests), proposed to diners for having fun during the meal;

- Avoid entering scientific or health information on the proposed food, because these info are regulated by the rules on public health. 


\section{Conclusions}

Narrative menus are tools containing evocative graphics, texts (flash fiction) connected to multimedia resources on the Web via smartphone, enabling customers to enjoy a convivial space and a better interaction with the food service.

This documentation, provided with explanatory notes (selected by documentalists and experts), creates an additional cultural value and promotes social exchanges with producers and chefs.

The smart menu here outlined can be considered a new form of "Food Magazine", even if it is not distributed through newspapers and books but through web services in restaurants, cafeterias and snack bars. The periodicity of publication may vary according to the characteristics of the restaurant. A suitable periodicity should be four numbers per year (spring, summer, autumn and winter collection), according to the restaurant seasonal food offering.

From time to time, some annexes (supernumerary pages inside the menu) can be loosely inserted between the standard menu pages, regarding tasting experience initiative or gastronomic novelties offered to customers.

Also new visual storytelling from producers or skilled food craftsmen, may be reported as annexes, to increase customer interest for the publication. Of course narrative menu should not represent a vanity press, but a direct and conscious commitment of people, telling their personal or familiar food stories, also because they may make this communication unique.

In our experience, the narrative menu has a real socialization value. There may be objections to a "disturbing" effect of this way of publishing, but, after all, some attention in the interaction with a smartphone in a loud dining room is required.

However, the menu fulfills all the functions of an ordinary, well-structured restaurant menu. It allows aware consumers, to access additional information on the quality and healthiness of the food they are about to eat, in a contextually appropriate topic.

Narrative menu don't require a long-lasting interaction, isolating customers from their friends, unless they want to use it expressly for this purpose (single customers or awaiting the arrival of a diner).

In this way, narratives that report the experiences of farmers, breeders and cooks, can come on stage, with an entertainment function for "room storytelling".

If the narrative menus were to take hold as true editorial channels (i.e. adopted by publishing houses, scientific magazines, catering companies), narratives like learning objects (narrative objects) would represent a sort of catalogue of transmedia food literature for smart consumers, an opportunity for e-commerce and great advantages for advanced restaurants.

\section{Acknowledgments}

I wish to thank the software house Goodmen.it Srl (Perugia, Italy) for the help and enthusiasm in discussing the structure of the Smart Narrative Menu project and the direction, room and kitchen teams of the "Valter a Solomeo" Restaurant (Solomeo, Corciano, Italy), for their collaboration in collecting data and experiences.

\section{References}

1. Selfa, T., Qazi, J. (2005). Place, Taste, or Face-to-Face? Understanding Producer-Consumer Networks in "Local" Food Systems in Washington State. Agriculture and Human Values, 22(4), 451464.

2. Kneafsey, M., Cox, R., Holloway, L., Dowler, E., Venn, L., Tuomainen, H. (2008). Reconnecting Consumers, Producers and Food: Exploring Alternatives. Publisher Berg, New York.

3. Papaoikonomou, E., Ginieis, M. (2017). Putting the farmer's face on food: governance and the producer-consumer relationship in local food systems. Agriculture and Human Values, 34:53-67. 
4. Kesarwani, A. (2019). E-Marketing Worked As an Effective Communication Between Consumer and Producer. Proc. 10th International Conference on Digital Strategies for Organizational Success, 4 January.

5. Ciappelloni, R. (2012). As we may print: The augmented wall newspapers". Sanità Pubblica Veterinaria, 74, October, http://indice.spvet.it\#559

6. Ciappelloni, R., Tabarrini, L. (2012). Communication activities for consumers in the food distribution network through storytellers and exhibitioners (Part One). Sanità Pubblica Veterinaria, 73, August, http://indice.spvet.it\#557

7. Ciappelloni, R. (2015). Giornali Murali "Smart" \& Poster "Aumentati". Un contributo per liberare il potenziale informativo degli Enti di ricerca pubblici - Smart wall newspapers \& augmented posters. A contribution to unleash the information potential of the public research Institutes. Sanità Pubblica Veterinaria, 88, February, http://spvet.it/indice-spv.html\#a619

8. Spvet.it. Manifesto del Gruppo di Lavoro per la Narrativa Biomedica [Approx. 0.3] (2009). Sanità Pubblica Veterinaria, 57, http://www.spvet.it/corrente/webzine/014.html

9. Ciappelloni, R., Nucci, D., Benedetti, A., Flammini, R., Falocci, G., Gaggioli, N., Montanucci, N., Heymann, A. J., Fruttini, L., Marini, C., Tabarrini, L. (2014). Bringing the Library 2.0 closer to the people: theatre storytelling, scientific lectures and nice food. Sanità Pubblica Veterinaria, 85 June. http://www.spvet.it/corrente/webzine/documenti/596.pdf, https://docplayer.net/41879133-14-theahil-conference.html

10. OVUD, Ospedale Veterinario Universitario Didattico, Università di Perugia - Didactic Veterinary Hospital, University of Perugia, Italy (2016). Smart poster initiative. http://spvet.it/archivio/numero95/628.html

11. Antun, J. (2005). Menu Analysis. Journal of Nutrition in Recipe \& Menu Development, 3(3/4), 81102.

12. Annaraud, K. (2008). Restaurant Menu Analysis Can We Go Further? Journal of Foodservice Business Research, 10(4), 25-37.

13. Long, M. W., Deirdre K. T., Cradock A. L., Batchelder, H., Gortmaker S. L. ( 2015). Systematic Review and Meta-analysis of the Impact of Restaurant Menu Calorie Labeling. American Journal of Public Health, 105, e11_e24.

14. Byrd, K, Almanza, B, Ghiselli, R. F., Behnke, C. Eicher-Miller, H. A. (2018). Adding sodium information to casual dining restaurant menus: Beneficial or detrimental for consumers? Appetite, 1 , $125: 474-485$.

15. Kraak, I. V. (2020). The US chain restaurant industry must transform its business model to market healthy menu items to Americans to reduce obesity and chronic disease risks. The Journal of Nutrition, 150(4), 656-657.

16. Noone, B. M., Cachia, G. (2020). Menu engineering re-engineered: Accounting for menu item substitutes in pricing and menu placement decisions. International Journal of Hospitality Management, 87, 102504.

17. Soh J, Sharma, A. (2021). The effect of menu presentation on consumers' willingness to purchase. Tourism and Hospitality Research, 1467358420987585.

18. Chavan, V., Jadhav, P., Korade, S., Teli, P. (2015). Implementing customizable online food ordering system using web based application. International Journal of Innovative Science, Engineering \& Technology, 2(4), 722-727.

19. Caroline, J., Fiorenza, E. L., Chakraborty, A., Rishi, R., Baghel, K. (2018). Smart Menu Card System. Proceedings of the 3rd International Conference on Communication and Electronics Systems, pp. 847-849.

20. Gunawardena, D., Sarathchandra, K. (2020). BestDish: A Digital Menu and Food Item Recommendation System for Restaurants in the Hotel Sector. International Conference on Image Processing and Robotics (ICIP), Negombo, Sri Lanka, 1-7.

21 Kurland, L. G., Gilbert, E. (1985). Integrated interactive restaurant communication system for food and entertainment processing. United States Patent. US4553222A.

22. Leung, X. Y., Josiam, B. M., Moody, B. M. (2020). "I'd like to order with a server" an experimental study of restaurant menu performance. Asia Pacific Journal of Tourism Research, 25(7), 766-779.

23. Van Rijswijk, W., Frever, L. J., (2012). Consumer needs and requirements for food and ingredient traceability information. International Journal of Consumer Studies, 36, 282-290.

24. Dover Press (2017). Franz Von Stuck - Of Menus and Mythology: Late Nineteenth-Century Print Graphics: Late Romantic Graphic Works. Publisher Dover Books USA. 
25. Heimann, J., Heller, S., Mariani, J. (2018). Menu design in America. Publisher Taschen GmbH, Germany.

26. Franklin, V., Johnson, A. (2019). Menus That Made History: Over 2000 Years of Menus from Ancient Egyptian Food for the Afterlife to Elvis Presley's Wedding Breakfast. Publisher Kyle Books. London.

27. Bolter, J. D. Grusin, R. (1999). Remediation: Understanding New Media. MIT Press, Cambridge. 